\title{
Shape-Based Diffeomorphic Registration on Hippocampal Surfaces Using Beltrami Holomorphic Flow
}

\author{
Lok Ming Lui ${ }^{1,2}$, Tsz Wai Wong ${ }^{2}$, Paul Thompson ${ }^{3}$, Tony Chan ${ }^{4}$, \\ Xianfeng $\mathrm{Gu}^{5}$, and Shing-Tung $\mathrm{Yau}^{1}$ \\ 1 Department of Mathematics, Harvard University, Cambridge, MA, USA \\ 2 Department of Mathematics, UCLA, Los Angeles, CA, USA \\ 3 Laboratory of Neuro Imaging, UCLA School of Medicine, Los Angeles, CA, USA \\ ${ }^{4}$ Hong Kong University of Science and Technology, Hong Kong \\ 5 Department of Computer Science, SUNY Stony Brook, Stony Brook, NY, USA
}

\begin{abstract}
We develop a new algorithm to automatically register hippocampal(HP) surfaces with complete geometric matching, avoiding the need to manually label landmark features. A good registration depends on a reasonable choice of shape energy that measures the dissimilarity between surfaces. In our work, we first propose a complete shape index using the Beltrami coefficient and curvatures, which measures subtle local differences. The proposed shape energy is zero if and only if two shapes are identical up to a rigid motion. We then seek the best surface registration by minimizing the shape energy. We propose a simple representation of surface diffeomorphisms using Beltrami coefficients, which simplifies the optimization process. We then iteratively minimize the shape energy using the proposed Beltrami Holomorphic flow (BHF) method. Experimental results on $212 \mathrm{HP}$ of normal and diseased (Alzheimer's disease) subjects show our proposed algorithm is effective in registering HP surfaces with complete geometric matching. The proposed shape energy can also capture local shape differences between HP for disease analysis.
\end{abstract}

\section{Introduction}

The hippocampus(HP) is an important subcortical structure of the human brain that plays a key role in long-term memory and spatial navigation. Surface-based shape analysis is commonly used to study local changes of HP surfaces due to pathologies such as Alzheimer disease (AD), schizophrenia and epilepsy[11. When comparing data on two anatomical surfaces, a 1-1 correspondence must be computed to register one surface nonlinearly onto the other. On HP surfaces, there are no well-defined anatomical landmark features that can be used as a constraint to establish good correspondences. High-field structural or functional imaging, where discrete cellular fields are evident [15], is still not routinely used. Finding meaningful registrations between HP surfaces becomes challenging. Inaccuracies in shape analysis are often introduced due to incorrect registrations. In fact, shape analysis and surface registration are closely related. The results of 


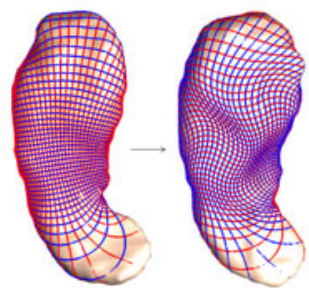

Initial surface map

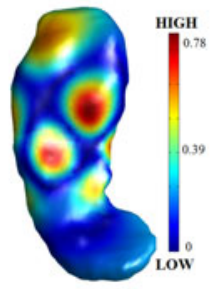

$\mathrm{BC}$ representation

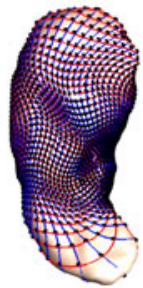

Reconstructed map

Fig. 1. Representation of surface registration using Beltrami Coefficients

shape analysis can be highly affected by the registration, but a good registration depends largely on the appropriate choice of shape measure that captures dissimilarities between surfaces. Therefore, it is of utmost importance to combine the two processes and define a suitable shape measure to drive the registration.

Here we developed an algorithm to automatically register HP surfaces with complete geometric matching, avoiding the need to manually label landmark features. We first propose a complete shape index using the Beltrami coefficient (BC) and curvatures, which measures subtle local differences. The shape energy is identically zero if and only if two shapes are equal up to a rigid motion. We then minimize the shape energy to obtain the best surface registration with complete geometric matching. We propose a simple representation of surface diffeomorphisms using BCs, which simplifies the optimization. We then optimize the shape energy using the Beltrami Holomorphic flow (BHF) method. The optimal shape energy obtained may also be used to measure local shape differences across subjects or time.

\section{Related Work}

Surface registration has been studied extensively. Conformal or quasi-conformal surface registration is commonly used 4514, and gives a parameterization minimizing angular distortions. However, it cannot guarantee the matching of geometric information such as curvature across subjects. Landmark-based diffeomorphisms are often used to compute, or adjust, cortical surface parameterizations 3612]. These methods provide good registrations when corresponding landmark points on the surfaces can be labeled in advance. It is, however, difficult for HP surfaces on which there are no well-defined anatomical landmarks. Some authors have proposed driving features into correspondence based on shape information. Lyttelton et al. 8] computed surface parameterizations that match surface curvature. Fischl et al. [1] improved the alignment of cortical folding patterns by minimizing the mean squared difference between the average convexity across a set of subjects and that of the individual. Wang et al. 13. computed surface registrations that maximize the mutual information between mean curvature and conformal factor maps across subjects. Lord et al. [7] matched surfaces by minimizing the deviation from isometry. The shape indices that drive the 
registration process in these approaches are not complete shape measurements and do not capture shape differences completely. There are cases when two different surfaces might have the same shape value. This could lead to inaccurate registration results.

\section{Theoretical Background and Definitions}

Given two Riemann surfaces $M$ and $N$, a map $f: M \rightarrow N$ is conformal if it preserves the surface metric up to a multiplicative factor. One generalization of conformal maps is the quasi-conformal maps, which are orientation-preserving homeomorphisms between Riemann surfaces with bounded conformality distortion, in the sense that their first order approximations takes small circles to small ellipses of bounded eccentricity [2]. Thus, a conformal homeomorphism that maps a small circle to a small circle may also be regarded as quasi-conformal. Mathematically, $f: \mathbb{C} \rightarrow \mathbb{C}$ is quasi-conformal if it satisfies the Beltrami equation: $\frac{\partial f}{\partial \bar{z}}=\mu(z) \frac{\partial f}{\partial z}$, for some complex valued function $\mu$ satisfying $\|\mu\|_{\infty}<1$. $\mu$ is called the Beltrami coefficient (BC), which is a measure of non-conformality. In particular, the map $f$ is conformal around a small neighborhood of $p$ when $\mu(p)=0$. From $\mu(p)$, we can determine the angles of the directions of maximal magnification and shrinking and the amount of them as well. Specifically, the angle of maximal magnification is $\arg (\mu(p)) / 2$ with magnifying factor $1+|\mu(p)|$; The angle of maximal shrinking is the orthogonal angle $(\arg (\mu(p))-\pi) / 2$ with shrinking factor $1-|\mu(p)|$. The distortion or dilation is given by: $K=(1+|\mu(p)|) /(1-|\mu(p)|)$.

\section{Proposed Model}

\subsection{A Complete Shape Index}

A good registration depends greatly on the appropriate choice of a shape measure to capture dissimilarities between surfaces. We propose a complete shape index $E_{\text {shape }}$ using the Beltrami coefficient and curvatures, which measures subtle local changes completely. Given two HP surfaces $S_{1}$ and $S_{2}$. Let $f: S_{1} \rightarrow S_{2}$ be a registration between $S_{1}$ and $S_{2}$. The complete shape index $E_{\text {shape }}$ is defined as follow: $E_{\text {shape }}(f)=\alpha|\mu|^{2}+\beta\left(H_{1}-H_{2}(f)\right)^{2}+\gamma\left(K_{1}-K_{2}(f)\right)^{2}$ where $\mu$ is the Beltrami coefficient of $f ; H_{1}, H_{2}$ are the mean curvatures on $S_{1}$ and $S_{2}$ respectively; and $K_{1}, K_{2}$ are the Gaussian curvatures. The first term measures the conformality distortion of the surface registration. The second and third terms measure the curvature mismatch. It turns out $E_{\text {shape }}$ is a complete shape index that measures subtle shape differences between two surfaces. It can be proven that $E_{\text {shape }}(f)=0$ if and only if $S_{1}$ and $S_{2}$ are equal up to a rigid motion. For HP shape analysis, it is good because clinically we are more interested in shape changes than their orientation. Also, by adjusting the parameters (i.e., $\alpha$, $\beta$ and $\gamma), E_{\text {shape }}$ can be made equivalent to other existing shape indices. For example, when $\beta=0, E_{\text {shape }}$ is equivalent to the isometric shape index; when 
$\alpha=0, E_{\text {shape }}$ is equivalent to the curvature index; when $\beta=\gamma=0, E_{\text {shape }}$ measures the conformality distortion. In our work, we set $\alpha=1$ and $\beta=\gamma=2$ to measure complete shape changes.

We can now minimize $E_{\text {shape }}$ to obtain the optimized surface map $\tilde{f}$ that best matches the geometry. One advantage of using $E_{\text {shape }}$ is that it can be defined in the space of BCs. The space of BCs is a simple functional space, which makes the optimization much easier.

\subsection{Surface Map Representation Using Beltrami Coefficients}

Surface registration is commonly parameterized using $3 \mathrm{D}$ coordinate functions in $\mathbb{R}^{3}$. This representation is difficult to manipulate. For example, the $3 \mathrm{D}$ coordinate functions have to satisfy certain constraints on the Jacobian $J$ (namely, $J>0$ ), to preserve the 1-1 correspondence of the surface maps. Enforcing this constraint adds extra difficulty in optimizing surface maps. The diffeomorphic property is often lost during the optimization. We propose a simple representation of surface diffeomorphisms using Beltrami coefficients (BCs). Fixing any 3 points on a pair of surfaces, there is a 1-1 correspondence between the set of surface diffeomorphisms between them and the set of BCs on the source domain.

Suppose $S_{1}$ and $S_{2}$ are both either genus 0 closed surfaces or simply connected open surfaces. $S_{1}$ and $S_{2}$ can be conformally parameterized with a global patch $D$ 414. Let $f: S_{1} \rightarrow S_{2}$, and given 3 point correspondences. In this work, we chose the 3 corresponding points based on the initial conformal registration. But we can easily generalize our method by incorporating a Mobius transformation that will help us to automatically detect optimal 3-point correspondences. Denote the parameterizations by $\phi_{1}: S_{1} \rightarrow D$ and $\phi_{2}: S_{2} \rightarrow D$. Now, we can compute the Beltrami coefficient $\mu_{f}$ associated uniquely to $f$ to represent $f$ (See Figure 1). The Beltrami coefficient $\mu_{f}$ can be computed by considering the composition map $\tilde{f}=\phi_{2} \circ f \circ \phi_{1}^{-1}: D \rightarrow D$. Mathematically, $\mu_{f}$ is given by the following formula: $\mu_{f}=\frac{\partial \tilde{f}}{\partial \bar{z}} / \frac{\partial \widetilde{f}}{\partial z}=\frac{1}{2}\left(\frac{\partial \tilde{f}}{\partial x}+\sqrt{-1} \frac{\partial \tilde{f}}{\partial y}\right) / \frac{1}{2}\left(\frac{\partial \tilde{f}}{\partial x}-\sqrt{-1} \frac{\partial \tilde{f}}{\partial y}\right)$.

The space of BCs is a simple functional space. There are no restrictions on $\mu$ that it has to be 1-1, surjective or satisfy some constraints on the Jacobian. Using the Beltrami representation makes the optimization process of surface maps much easier.

\subsection{Optimized Surface Registration Matching the Geometry}

$E_{\text {shape }}$ gives us a complete shape index which measures local dissimilarities between two surfaces. Specifically, $E_{\text {shape }}(f)=0$ if and only if $S_{1}$ and $S_{2}$ are equal up to a rigid motion. Therefore, the surface map $f$ minimizing $E_{\text {shape }}(f)$ is the best registration that best matches the geometric information. Given two HP surfaces $S_{1}$ and $S_{2}$. We propose to find $f: S_{1} \rightarrow S_{2}$ that minimizes $E=\int E_{\text {Shape }}(f)$. To simplify the computation, we can conformally parameterize $S_{1}$ and $S_{2}$ onto the parameter domain $D$. So, all computations are carried out on the simple domain $D$. By representing surface maps with Beltrami coefficients $\mu$, 


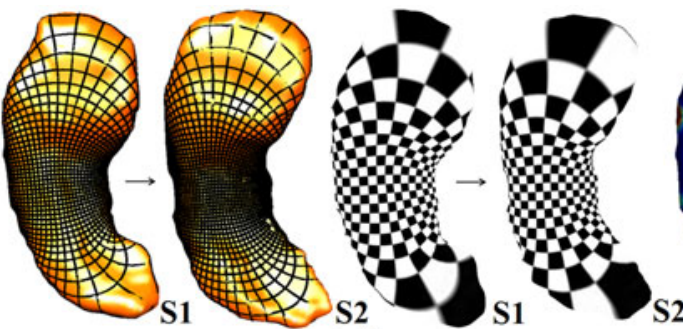

(A)

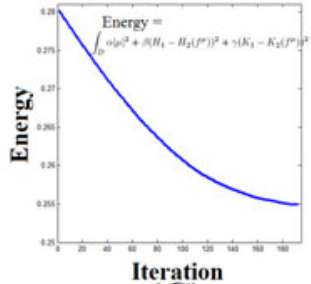

(C)

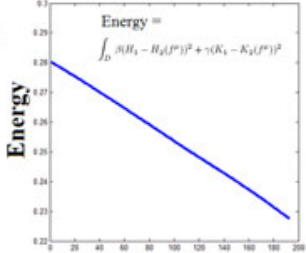

nenation

(D)

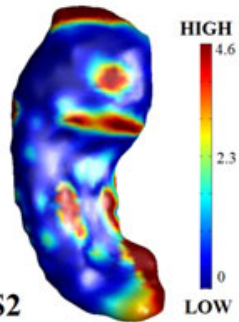

(B)

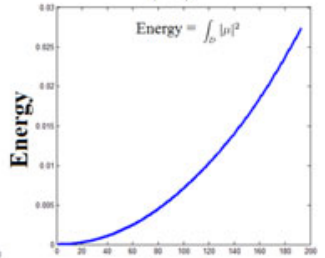

Iteration

(E)

Fig. 2. Shape registration with geometric matching using Beltrami Holomorphic flow

we can define the energy on the space of BCs - a much simpler functional space for the optimization process. Mathematically, the compound energy $E$ can be written with respect to $\mu$ as: $E(\mu)=\int_{D} \alpha|\mu|^{2}+\beta\left(H_{1}-H_{2}\left(f^{\mu}\right)\right)^{2}+\gamma\left(K_{1}-K_{2}\left(f^{\mu}\right)\right)^{2}$. The variation of $f^{\mu}$ under the variation of $\mu$ can be expressed explicitly. Suppose $\widetilde{\mu}(z)=\mu(z)+t \nu(z)+\mathcal{O}\left(t^{2}\right)$. Then, $f^{\widetilde{\mu}(z)}(w)=f^{\mu}(w)+t V\left(f^{\mu}, \nu\right)(w)+\mathcal{O}\left(t^{2}\right)$, where $V\left(f^{\mu}, \nu\right)(w)=-\frac{f^{\mu}(w)\left(f^{\mu}(w)-1\right)}{\pi} \int_{D} \frac{\nu(z)\left(f_{z}^{\mu}(z)\right)^{2} d x d y}{f^{\mu}(z)\left(f^{\mu}(z)-1\right)\left(f^{\mu}(z)-f^{\mu}(w)\right)}$ Using the variational formula, we can derive the Euler-Lagrange equation of $E(\mu)$ easily. Specifically, we can minimize $E(\mu)$ by the following iterative scheme:

$\mu^{n+1}-\mu^{n}=-2\left(\alpha \mu^{n}-\int_{z}\left[\left(\beta \widetilde{H}^{n}+\gamma \widetilde{K}^{n}\right) \cdot G^{n}, \operatorname{det}\left(\beta \widetilde{H}^{n}+\gamma \widetilde{K}^{n}, G^{n}\right)\right]\right) d t$, where $\int_{w} \bullet:=\int_{D} \bullet d w$ and $\int_{z} \bullet:=\int_{D} \bullet d z$ is defined as the integral over the variable $w$ and $z$ respectively; $\widetilde{H}:=\left(H_{1}-H_{2}\left(f^{\mu}\right)\right) \nabla H_{2}\left(f^{\mu}\right) ; \widetilde{K}:=\left(K_{1}-\right.$ $\left.K_{2}\left(f^{\mu}\right)\right) \nabla K_{2}\left(f^{\mu}\right)$; $\operatorname{det}(a, b)$ is the determinant of the 2 by 2 matrix or equivalently, the norm of the cross product of $a$ and $b$.

We call this iterative algorithm the Beltrami Holomorphic flow (BHF). Note that starting with a conformal map with $\mu=0$, the first term of the energy ensures $\mu$ to satisfy $\|\mu\|_{\infty}<1$. Hence, during the BHF process, the maps are guaranteed to be diffeomorphic and are holomorphic in $t$.

\section{$5 \quad$ Experimental Results}

We tested our algorithm on $212 \mathrm{HP}$ surfaces automatically extracted from 3D brain MRI scans with a validated algorithm 9]. Scans were acquired from normal and diseased (AD) elderly subjects at $1.5 \mathrm{~T}$ (on a GE Signa scanner). Experiments have been carried out on a laptop with a $2.4 \mathrm{GHz}$ DUO CPU. The 


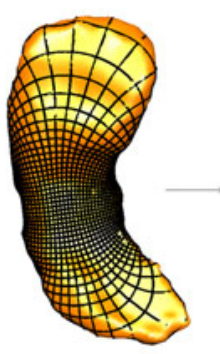

Normal 1

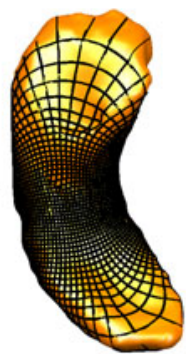

Normal 2

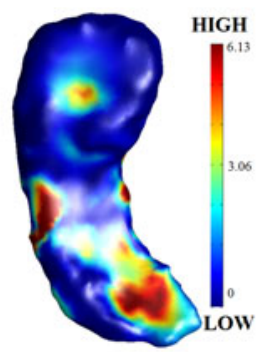

Shape index

Fig. 3. BHF registration between two normal subjects. The shape index $E_{\text {shape }}$ is plotted on the right, which captures local shape differences.

algorithm takes about 4-5 minutes to compute a registration between meshes with $40 \mathrm{~K}$ vertices.

Figure 1 shows the Beltrami representations of bijective surface maps. The left column shows a bijective surface map between the HP surfaces. The middle column shows the Beltrami (BC) representations of the maps. The right column shows the reconstruction of surface maps from their BCs. The reconstructed maps closely resemble the original maps, meaning that BCs can effectively represent bijective surface maps. Figure 2(A) shows two different HP surfaces. They are registered using our proposed $\mathrm{BHF}$ algorithm with geometric matching. The registration is visualized using a grid map and texture map, which shows a smooth 1-1 correspondence. The optimal shape index $E_{\text {shape }}$ is plotted as colormap in (B). E shape effectively captures the local shape difference between the surfaces. (C) shows the shape energy in each iteration. With the BHF algorithm, the shape energy decreases as the number of iterations increases. (D) shows the curvature mismatch energy $\left(E=\int \beta\left(H_{1}-H_{2}(f)\right)^{2}+\gamma\left(K_{1}-K_{2}(f)\right)^{2}\right)$. It decreases as the number of iterations increases, meaning that the geometric matching improves. (E) shows the Beltrami coefficient of the map in each iteration, which shows the conformality distortion of the map. Some conformality is intentionally lost to allow better geometric matching.

Figure 3 shows the BHF registration between two normal HPs. The complete shape index $E_{\text {shape }}$ is plotted as colormap on the right. Again, $E_{\text {shape }}$ can accurately capture local shape differences between the normal HP surfaces.

Figure 4 shows the BHF hippocampal registrations between normal elderly subjects and subjects with Alzheimer's disease. The BHF registrations give smooth 1-1 correspondences between the HP surfaces. We can use the complete shape index $E_{\text {shape }}$ to detect local shape differences between healthy and unhealthy subjects.

We also study the temporal shape changes of normal and AD HP surfaces, as shown in Figure 5. For each subject, we compute the deformation pattern of its HP surfaces measured at time $=0$ and time $=12$ Months (see [10] for longitudinal scanning details). The left two panels show the temporal deformation patterns for two normal subjects. The middle two panels show the temporal 


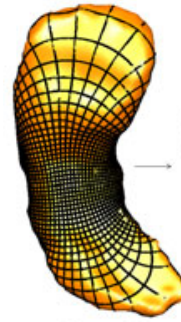

Normal

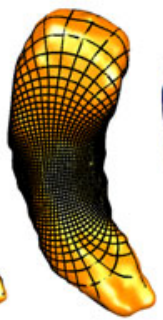

Alzheimer 1

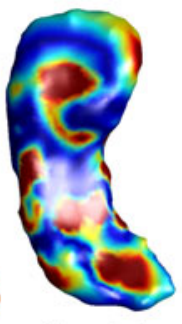

Shape index

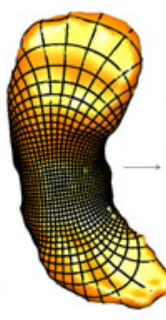

Normal

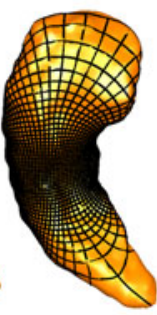

Alzheimer 2

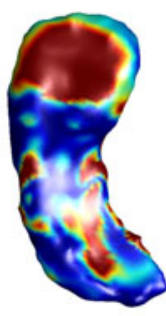

Shape index

Fig. 4. BHF registration between normal subjects and subjects with Alzheimer's disease. Their local shape differences are captured by $E_{\text {shape }}$.

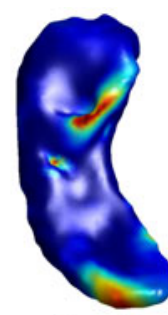

Normal 1

Normal $1 \quad$ Normal 2

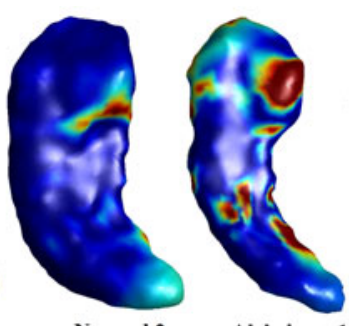

Alzheimer 1 Alzheimer 2 (from $t=0$ to $t=12$ Months)

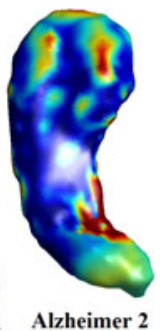

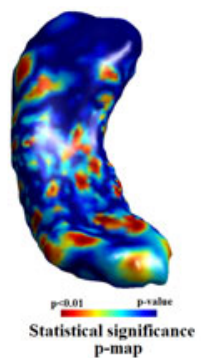

p-map

Fig. 5. Temporal hippocampal shape changes of normal and subjects with Alzheimer's disease

deformation patterns for two AD subjects. The last column shows the statistical significance p-map measuring the difference in the deformation pattern between the normal $(n=47)$ and AD $(n=53)$ groups, plotted on a control HP. The deep red color highlights regions of significant statistical difference. This method can be potentially used to study factors that influence brain changes in AD.

\section{Conclusion and Future Work}

We developed an algorithm to automatically register HP surfaces with complete geometric matching, avoiding the need for manually-labeled landmark features. We did this by defining a complete shape index to drive the registration. Experimental results on $212 \mathrm{HP}$ surfaces from normal and diseased(AD) subjects show our proposed algorithm is effective in registering HP surfaces over time and across subjects, with complete geometric matching. The proposed shape energy can also capture local shape differences between HPs for disease analysis. In future, we will use the BHF algorithm to systematically study the local shape differences and factors that affect deformation patterns between normal and AD subjects.

Acknowledgement. PT is supported by NIH: EB008432, EB008281, EB007813, HD050735 \& AG036535. TF is supported by NSF GEO-0610079, NIH 
U54 RR021813 and ONR N00014-09-1-0105. XG is supported by NIH 1R01EB0075300A1, NSF IIS 0916286, CCF0916235, CCF0830550, III0713145, and ONR N000140910228.

\section{References}

1. Fischl, B., et al.: High-resolution intersubject averaging and a coordinate system for the cortical surface. Human Brain Mapping 8, 272-284 (1999)

2. Gardiner, F., et al.: Quasiconformal Teichmuller Theory. Amer. Math. Soc., Providence (2000)

3. Glaunés, J., et al.: Landmark matching via large deformation diffeomorphisms on the sphere. J. Maths. Imaging and Vision 20, 179-200 (2004)

4. $\mathrm{Gu}, \mathrm{X}$., et al.: Genus zero surface conformal mapping and its application to brain surface mapping. IEEE Transactions on Medical Imaging 23(8), 949-958 (2004)

5. Hurdal, M.K., et al.: Discrete conformal methods for cortical brain flattening. Neuroimage 45, 86-98 (2009)

6. Leow, A., et al.: Brain structural mapping using a novel hybrid implicit/explicit framework based on the level-set method. NeuroImage 24(3), 910-927 (2005)

7. Lord, N.A., et al.: Simultaneous registration and parcellation of bilateral hippocampal surface pairs for local asymmetry quantification. IEEE Transactions on medical imaging 26(4), 471-478 (2007)

8. Lyttelton, O., et al.: An unbiased iterative group registration template for cortical surface analysis. NeuroImage 34, 1535-1544 (2007)

9. Morra, J., et al.: Validation of a fully automated 3d hippocampal segmentation method using subjects with alzheimer's disease, mild cognitive impairment, and elderly controls. Neuroimage 43(1), 59-68 (2008)

10. Morra, J., et al.: Automated mapping of hippocampal atrophy in 1-year repeat mri data in 490 subjects with alzheimer's disease, mild cognitive impairment, and elderly controls. Neuroimage, 45(1), S3-S15 (2009)

11. Thompson, P., et al.: Mapping hippocampal and ventricular change in alzheimer's disease. NeuroImage 22(4), 1754-1766 (2004)

12. Thompson, P., et al.: A surface-based technique for warping 3-dimensional images of the brain. IEEE Transactions on Medical Imaging 15(4), 1-16 (1996)

13. Wang, Y., et al.: Automated surface matching using mutual information applied to Riemann surface structures. In: Duncan, J.S., Gerig, G. (eds.) MICCAI 2005. LNCS, vol. 3750, pp. 666-674. Springer, Heidelberg (2005)

14. Wang, Y., et al.: Brain surface conformal parameterization using Riemann surface structure. IEEE Transactions on Medical Imaging 26(6), 853-865 (2007)

15. Zeineh, M., et al.: Dynamics of the hippocampus during encoding and retrieval of face-name pairs. NeuroImage 299(5606), 577-580 (2003) 